\title{
A TUNABLE QUADRATURE OSCILLATOR WITH ONLY TRANSCONDUCTANCE ELEMENTS AND GROUNDED CAPACITORS
}

\author{
IQBAL A. KHAN ${ }^{\mathrm{a}, *}$, MUSLIM T. AHMED ${ }^{\mathrm{b}}$ and NIGAR MINHAJ ${ }^{\mathrm{c}}$ \\ ${ }^{a}$ Department of Electronics Engineering, Aligarh Muslim University, Aligarh 202 002, India; \\ ${ }^{\mathrm{b}}$ Department of Electrical and Electronics Engineering, University of Bahrain, Isa Town, Bahrain; \\ ${ }^{\mathrm{c}}$ Electronics Engineering Section, Women's Polytechnic, Aligarh Muslim University, \\ Aligarh 202 002, India
}

(Received 18 May 2002; In final form 3 July 2002)

\begin{abstract}
A novel quadrature oscillator is realised with only transconductance elements (TEs) and grounded capacitors. The realised quadrature oscillator uses only two inverting and two non-inverting transconductance elements, along with two grounded capacitors. The quadrature oscillator enjoys the attractive features of low component count, independent frequency tuning and suitability for integration in MOS technology.
\end{abstract}

Keywords: Active networks, Transconductance element; $g_{m}-\mathrm{C}$ oscillators

\section{INTRODUCTION}

Recently, the transconductance elements and grounded capacitor (TGC)-based circuits have become very popular in the design of active networks [1-3]. The TGC circuits possesses superior high frequency performance and economised chip area requirement as compared with operational transconductance amplifier (OTA)-based circuits, along with its MOS compatibility for monolithic implementation [4-10].

In this paper a novel quadrature oscillator (QOC) is given which uses only transconductance elements and grounded capacitors. The resulting QOC is in the TGC form. The QOC enjoys independent frequency control through gate voltage $\left(V_{G}\right)$. The QOC was designed and verified using PSPICE-simulation.

\section{CIRCUIT REALISATION}

The inverting linear transconductance element (ITE) is shown in Figure 1, along with its CMOS implementation, symbol and circuit model [1, 2]. The non-inverting transconductance element (NTE) realisation and symbol is shown in Figure 2. The proposed TGC quadrature

*Corresponding author.

ISSN 0882-7516 print; ISSN 1563-5031 online (C) 2002 Taylor \& Francis Ltd

DOI: $10.1080 / 0882751021000051659$ 


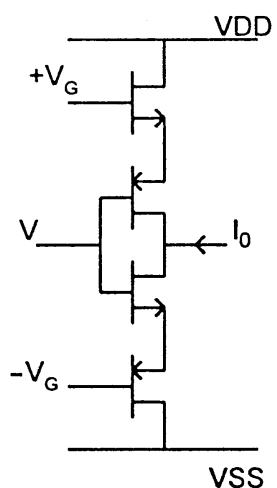

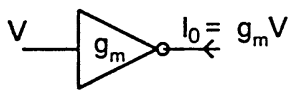

(b)
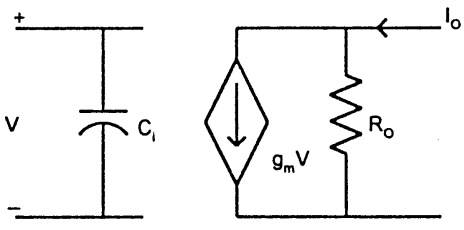

(C)

(a)

FIGURE 1 Inverting transconductance element (a) CMOS implementation; (b) Symbolic representation; (c) Circuit model.

oscillator using ITE and NTE is shown in Figure 3(a). It is basically realised by cascading an ideal integrator with an adjustable-pole inverting integrator in a closed loop configuration. The gain of the two integrator-loop of Figure 3(a) can be expressed as

$$
\frac{V_{3}}{V_{1}}=-\frac{g_{m 1} / C_{1}}{s+\left(g_{m 2}-g_{m 3}\right) / C_{1}} \cdot \frac{g_{m 4} / C_{2}}{s}
$$

The characteristic equation from (1) is

$$
s^{2}+s \frac{\left(g_{m 2}-g_{m 3}\right)}{C_{1}}+\frac{g_{m 1} g_{m 4}}{C_{1} C_{2}}=0
$$

which gives the condition of oscillation as

$$
g_{m 2} \leq g_{m 3}
$$

and the frequency of oscillation as

$$
\omega_{o}=\left(\frac{g_{m 1} g_{m 4}}{C_{1} C_{2}}\right)^{1 / 2}
$$

With $g_{m 1}=g_{m 2}=g_{m}$ and $C_{1}=C_{2}=C$, the frequency of oscillation can be expressed as

$$
\omega_{o}=\frac{g_{m}}{C}
$$

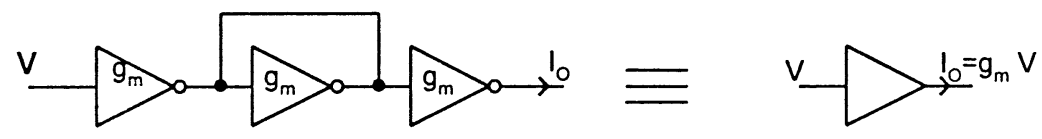

FIGURE 2 Non-inverting transconductance element. 


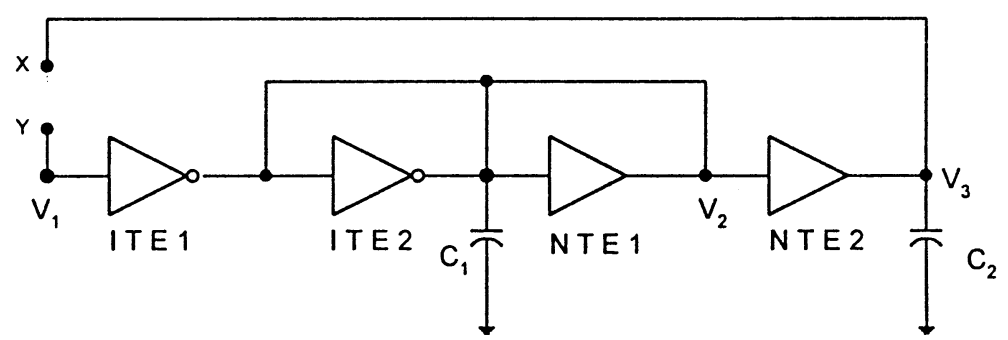

(a)

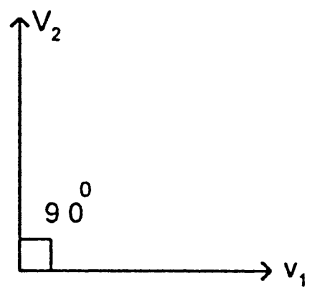

(b)

FIGURE 3 (a) TGC-quadrature oscillator; (b) Phasor diagram.

From (3) and (4) it is obvious that the QOC of Figure 3(a) can be set to oscillate through $g_{m 2}$ and $g_{m 3}$, while the frequency of oscillations can be controlled through $g_{m 1}$ and $g_{m 4}$. Thus it provides non-interactive tuning of the frequency of oscillation. At oscillating frequency

$$
\left.V_{1}\right|_{s=j \omega}=-j \alpha V_{2}, \quad \text { where } \alpha=\frac{g_{m}}{\omega C}
$$

Equation (6) depicts the quadrature outputs of the oscillator as shown in Figure 3(b).

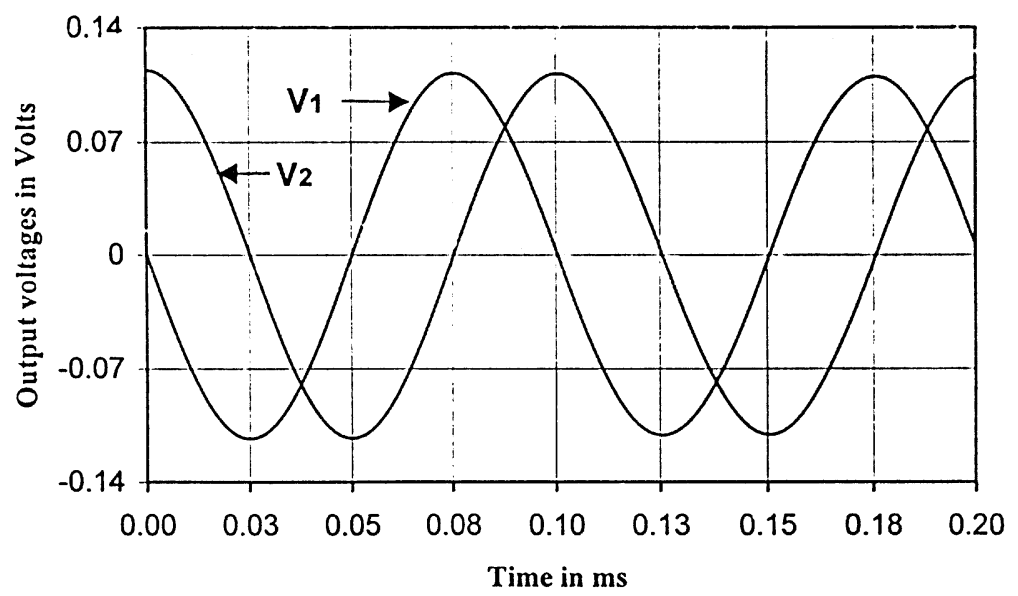

(a)

FIGURE 4 (a) Simulation-results of TGC quadrature oscillator of Figure 3(a). 


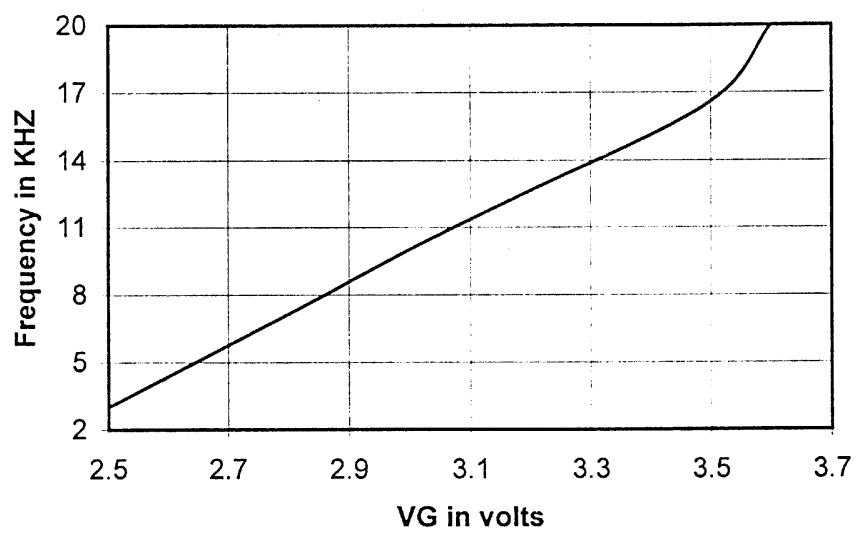

(b)

FIGURE 4 (b) Frequency tuning with gate voltage $V_{4}$.

\section{SIMULATION RESULTS}

To verify performance, the QOC of Figure 3(a) was simulated by PSPICE using the CMOS transconductance element of Figure 1(a) with CMOS model parameters given in reference [2]. Initially the QOC of Figure 3(a) (with nodes $X$ and $Y$ shorted) was designed with gate voltage $V_{G}= \pm 3 \mathrm{~V}$ (for which the transconductance value is $62 \mu \mathrm{mho}$ ), the $C_{1}=C_{2}=0.986 \mathrm{NF}$ for $f_{o}=10 \mathrm{kHz}$. The condition of oscillation was set by controlling the gate voltage of ITE2. The waveshapes obtained are shown in Figure 4(a), which clearly shows the quadrature outputs and thus verify the theory. The frequency of oscillation was controlled by gate voltage $V_{G}$ of ITE1 and NTE2 of the QOC. The variation of frequency of oscillation with $V_{G}$ is shown in Figure 4(b), which is linear within $17 \mathrm{kHz}$.

\section{CONCLUSION}

A novel quadrature oscillator with only transconductance elements and grounded capacitors is given. The given oscillator uses low component count and provides non-interactive tuning of its frequency of oscillation through gate voltage control. The quadrature oscillator is attractive for monolithic implementation in CMOS technology. The simulation results on the oscillator verify the theory.

\section{References}

[1] Tan, M. A. and Schaumann, R. (1988). Design of general biquadratic filters with only transconductances and grounded capacitors. I.E.E.E. Trans., CAS-35, 478-480.

[2] Tan, M. A., and Schaumann, R. (1989). Simulating general parameter LC-ladder filters for monolithic realisation with only transconductance elements and grounded capacitors. I.E.E.E. Transactions on Circuits and Systems, 36, 299-307.

[3] Schaumann, R. (1989). Design of continuous-time fully integrated filters: a review. IEE Proceedings of the Institution of Electrical Engineers, Pt. G, 136, 184-190.

[4] Sun, Y. and Flider, J. K. (1995). Resonator based universal OTA-grounded capacitor-filter. International Journal of Circuit Theory and Applications, 23, 261-265.

[5] Sun, Y. and Flider, J. K. (1997). Structure generation and design of multiple loop feedback OTA-grounded capacitor filters. I.E.E.E. Transactions on Circuits and Systems, 44, 1-11. 
[6] Efthivoulidis, G., Toth, L. and Tsividis, Y. P. (1998). Noise in $\mathrm{g}_{\mathrm{m}}-$ C filters. I.E.E.E. Transactions on Circuits and Systems-II: Analog and Digital Signal Processing, 45(3), 295-302.

[7] Sun, Y. (1998). OTA-C filter design using inductor substitution and bruton transformation methods. Electronics Letters, 34(22), 2082-2083.

[8] Khan, I. A., Ahmed, M. T. and Minhaj, N. (1992). Novel technique for immittance simulátion-realisation of some all-active simulators. Int. J. Electronics, 72(3), 431-441.

[9] Khan, I. A., Ahmed, M. T. and Minhaj, N. (1997). On transconductance-C quadrature oscillators. Int. J. of Electronics, 83, 201-207.

[10] Khan, I. A., Ahmed, M. T. and Minhaj, N. (1992). Tunable OTA-based multiphase sinusodial oscillators, Int. $J$. of Electronics, 72, 443-450. 

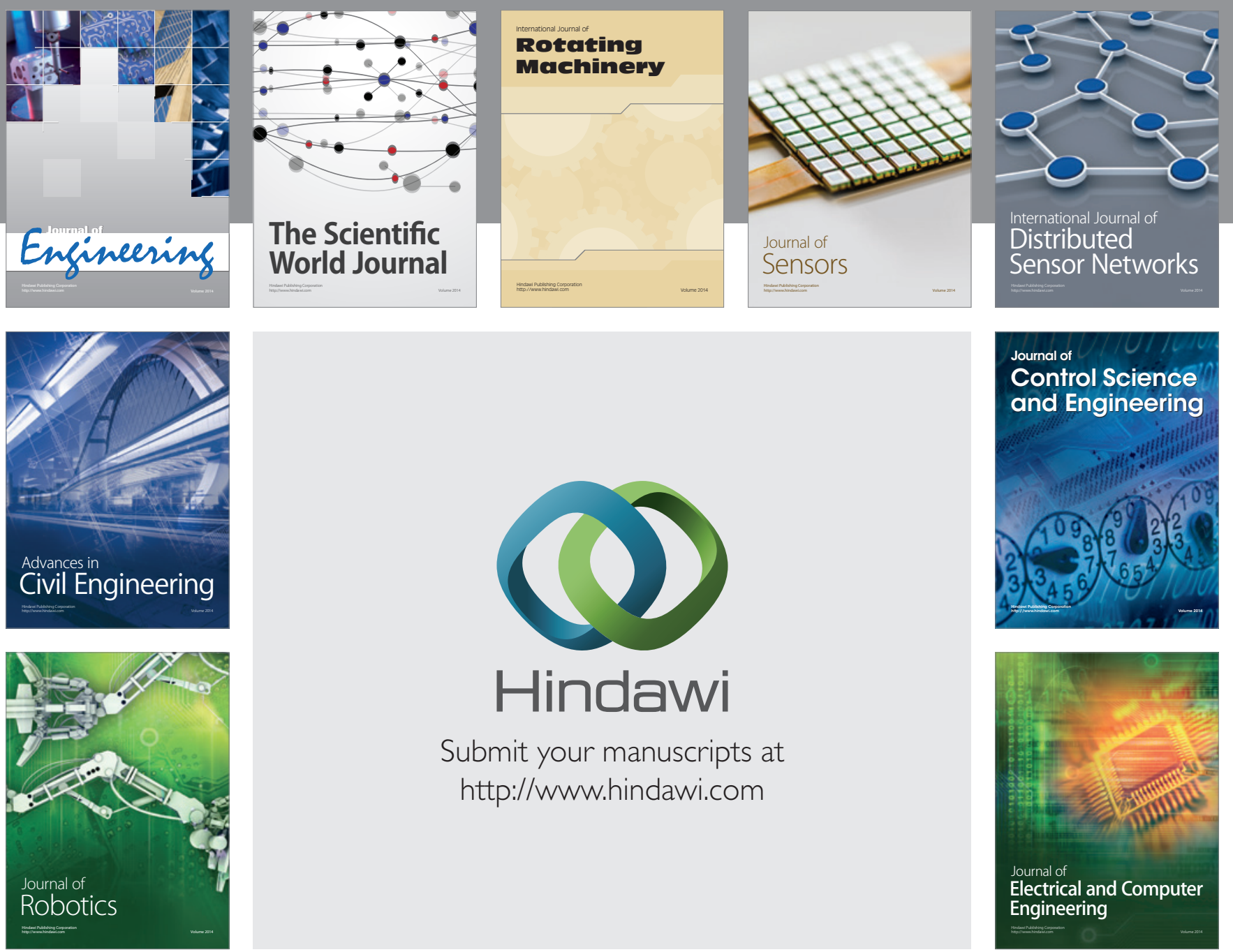

Submit your manuscripts at

http://www.hindawi.com
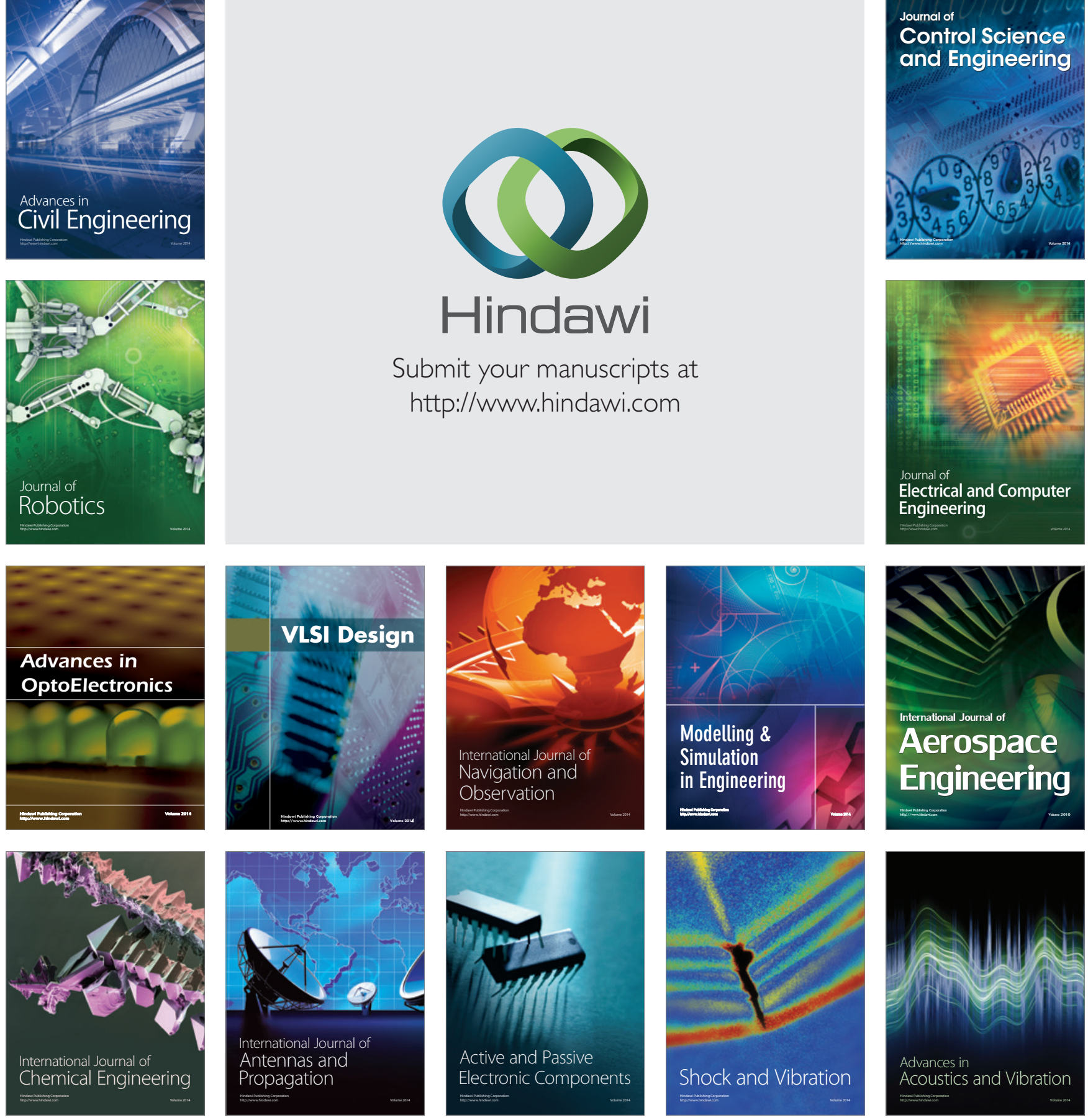